\title{
Stereoselective Synthesis of 1,4-Bifunctional Compounds by Regioselective Pd-Catalyzed Allylic Substitution Reaction
}

Naoyoshi Maezaki, Yuki Hirose, and Tetsuaki Tanaka*

Supporting Information 


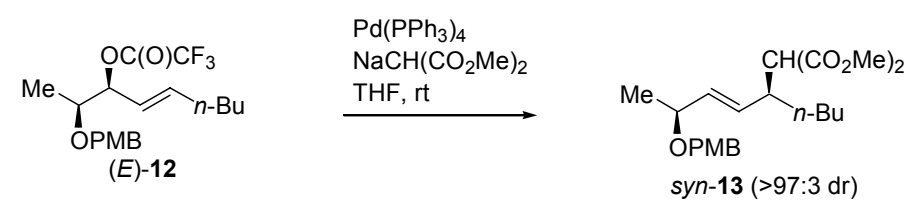

(a) with $\mathrm{NaCH}\left(\mathrm{CO}_{2} \mathrm{Me}\right)_{2}$ (Synthesis of Compound syn-13): $\mathrm{NaH}$ (60\% in oil, $\left.12.0 \mathrm{mg}, 0.300 \mathrm{mmol}\right)$ was washed $n$-hexane under $\mathrm{N}_{2}$ and suspended in THF $(2 \mathrm{~mL})$. Dimethyl malonate (38 $\left.\mu \mathrm{L}, 0.33 \mathrm{mmol}\right)$ was added dropwise to the suspension with stirring at $0{ }^{\circ} \mathrm{C}$ and the stirring was continued for $10 \mathrm{~min}$ at $\mathrm{rt}$. The solution of dimethyl sodiomalonate in THF was added to a mixture of $(E)-12(56.2 \mathrm{mg}, 0.15 \mathrm{mmol})$ and $\mathrm{Pd}\left(\mathrm{PPh}_{3}\right)_{4}(17.3 \mathrm{mg}, 0.015 \mathrm{mmol})$ in $\mathrm{THF}(2 \mathrm{~mL})$ and the whole was stirred at $\mathrm{rt}$ for $15 \mathrm{~min}$. The reaction was quenched with water and THF was evaporated. The residue was extracted with AcOEt. The extract was washed with brine prior to drying and solvent evaporation. The residue was chromatographed on silica gel with hexane-AcOEt (4:1) to give syn-13 (56.3 mg, 96\%) as a colorless oil.

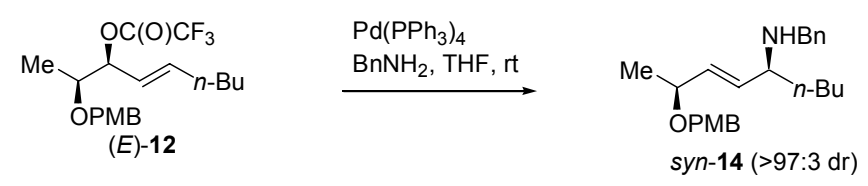

(b) with Amine (Synthesis of Compound syn-14): Benzylamine (33 $\mu \mathrm{L}, 0.30 \mathrm{mmol})$ was added to a mixture of $(E)-12$ $(56.2 \mathrm{mg}, 0.15 \mathrm{mmol})$ and $\mathrm{Pd}\left(\mathrm{PPh}_{3}\right)_{4}(17.3 \mathrm{mg}, 0.015 \mathrm{mmol})$ in $\mathrm{THF}(3 \mathrm{~mL})$ and the whole was stirred at $\mathrm{rt}$ for $30 \mathrm{~min}$. The reaction was quenched with water and THF was evaporated. The residue was extracted with AcOEt. The extract was washed with brine prior to drying and solvent evaporation. The residue was chromatographed on silica gel with hexane-AcOEt (4:1 to $2: 1)$ to give syn-14 (46.5 $\mathrm{mg}, 84 \%)$ as a colorless oil.

\section{Specific Rotations of New Chiral Compounds}

anti-2 $[\alpha]^{19} \mathrm{D}+8.61\left(c 1.13, \mathrm{CHCl}_{3}\right)$

anti-3 $[\alpha]^{22}-31.9\left(c 1.08, \mathrm{CHCl}_{3}\right)$

anti-5 $[\alpha]^{24}-30.0\left(c 0.95, \mathrm{CHCl}_{3}\right)$

anti-6 $[\alpha]^{24}{ }_{\mathrm{D}}-1.14\left(c 0.54, \mathrm{CHCl}_{3}\right)$

anti-8 $[\alpha]_{\mathrm{D}}^{25}+21.2\left(c 0.34, \mathrm{CHCl}_{3}\right)$

syn-7 $[\alpha]^{22}{ }_{D}-28.1\left(c 0.45, \mathrm{CHCl}_{3}\right)$

syn-11 $[\alpha]_{D}^{22}+49.5\left(c 0.66, \mathrm{CHCl}_{3}\right)$

(Z)-12 $[\alpha]^{25}-18.4\left(c\right.$ 1.07, $\left.\mathrm{CHCl}_{3}\right)$

syn-14 $[\alpha]^{23}{ }_{D}-67.1\left(c 0.57, \mathrm{CHCl}_{3}\right)$

anti-14 $[\alpha]^{23}{ }_{\mathrm{D}}-25.6\left(c 0.71, \mathrm{CHCl}_{3}\right)$ syn-2 $[\alpha]_{\mathrm{D}}^{25}+23.2\left(c\right.$ 1.03, $\left.\mathrm{CHCl}_{3}\right)$

anti-4 $[\alpha]^{23}{ }_{\mathrm{D}}-8.95\left(c 0.90, \mathrm{CHCl}_{3}\right)$

syn-5 $[\alpha]^{25}+33.3\left(c 1.01, \mathrm{CHCl}_{3}\right)$

anti-7 $[\alpha]^{23}{ }_{\mathrm{D}}+10.8\left(c 0.76, \mathrm{CHCl}_{3}\right)$

syn-6 $[\alpha]^{23}{ }_{D}-8.02\left(c 1.03, \mathrm{CHCl}_{3}\right)$

syn-8 $[\alpha]^{25}-42.6\left(c 0.68, \mathrm{CHCl}_{3}\right)$

(E)-12 $[\alpha]^{24}+23.8\left(c 0.82, \mathrm{CHCl}_{3}\right)$

syn-13 $[\alpha]^{23}{ }_{D}-46.9\left(c 0.76, \mathrm{CHCl}_{3}\right)$

anti-13 $[\alpha]^{24}-31.2\left(c 1.23, \mathrm{CHCl}_{3}\right)$ 


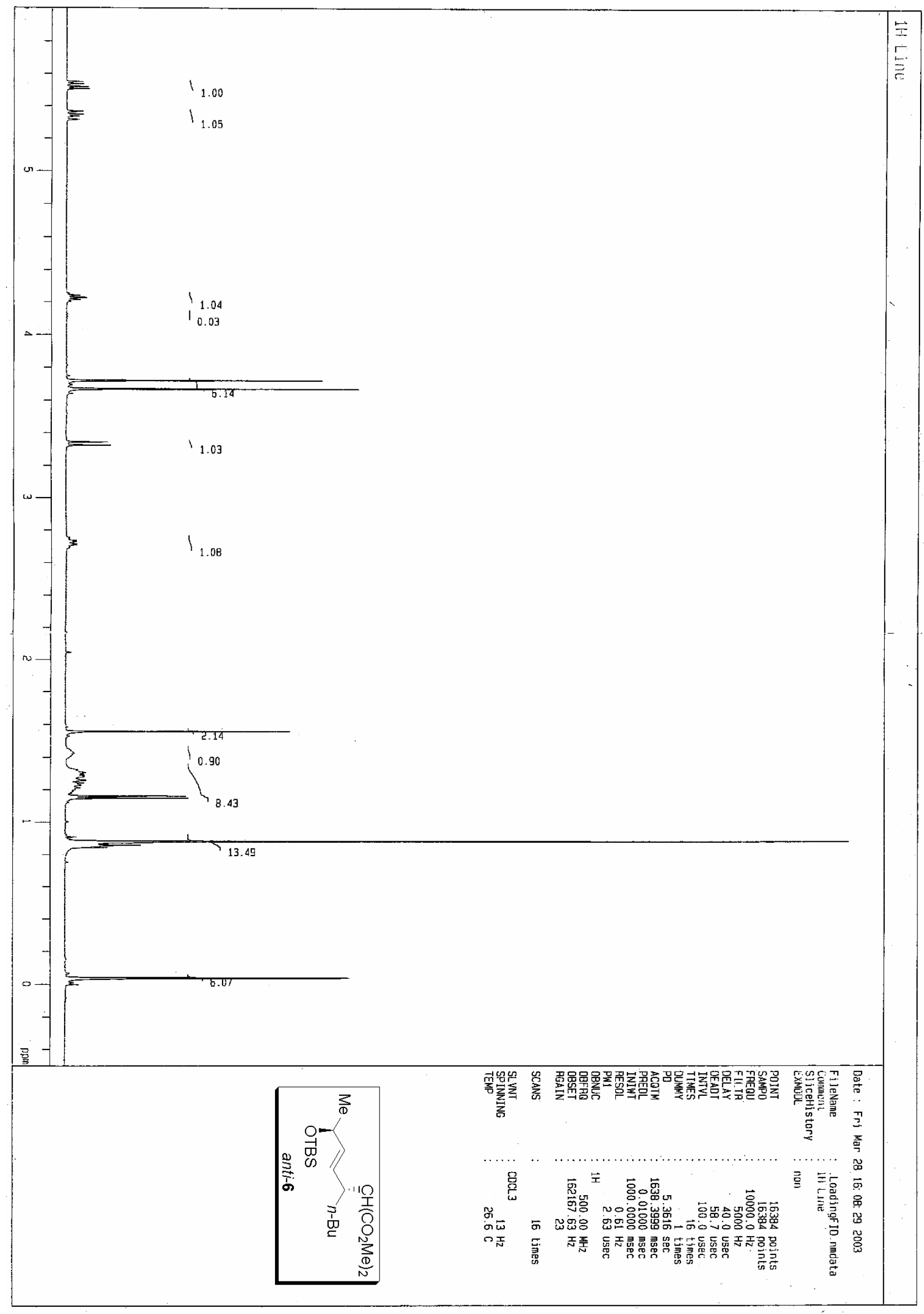




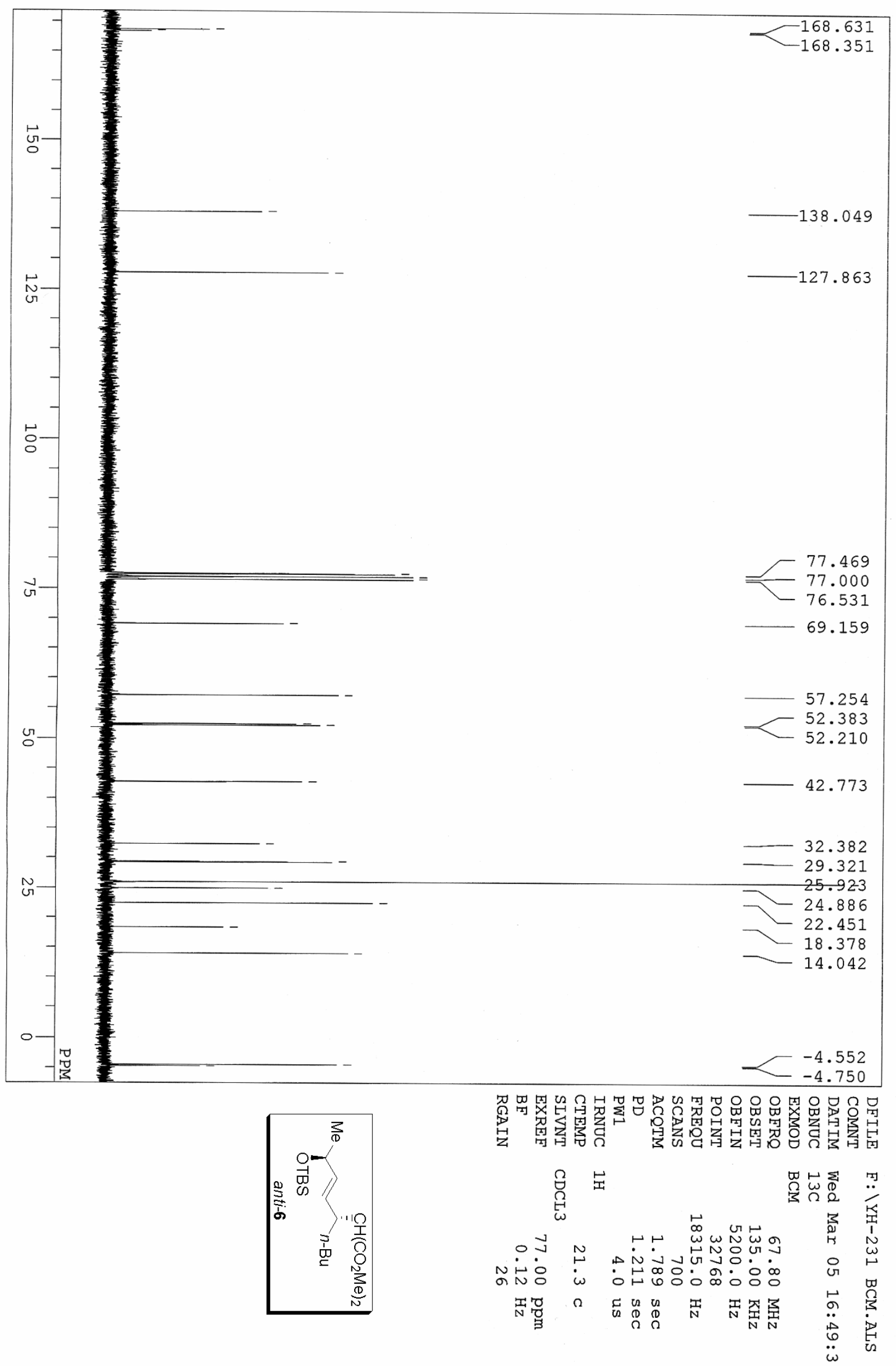




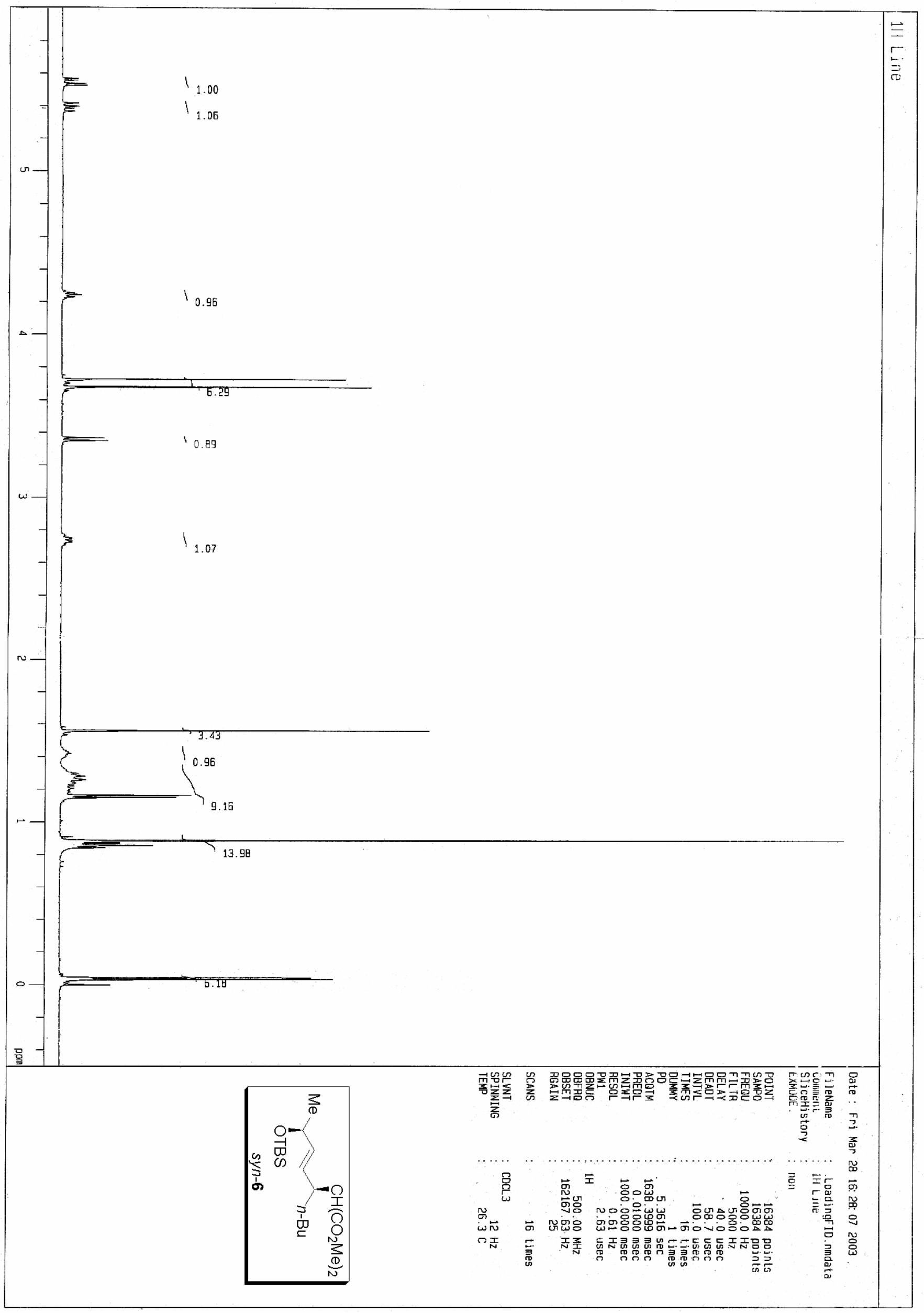



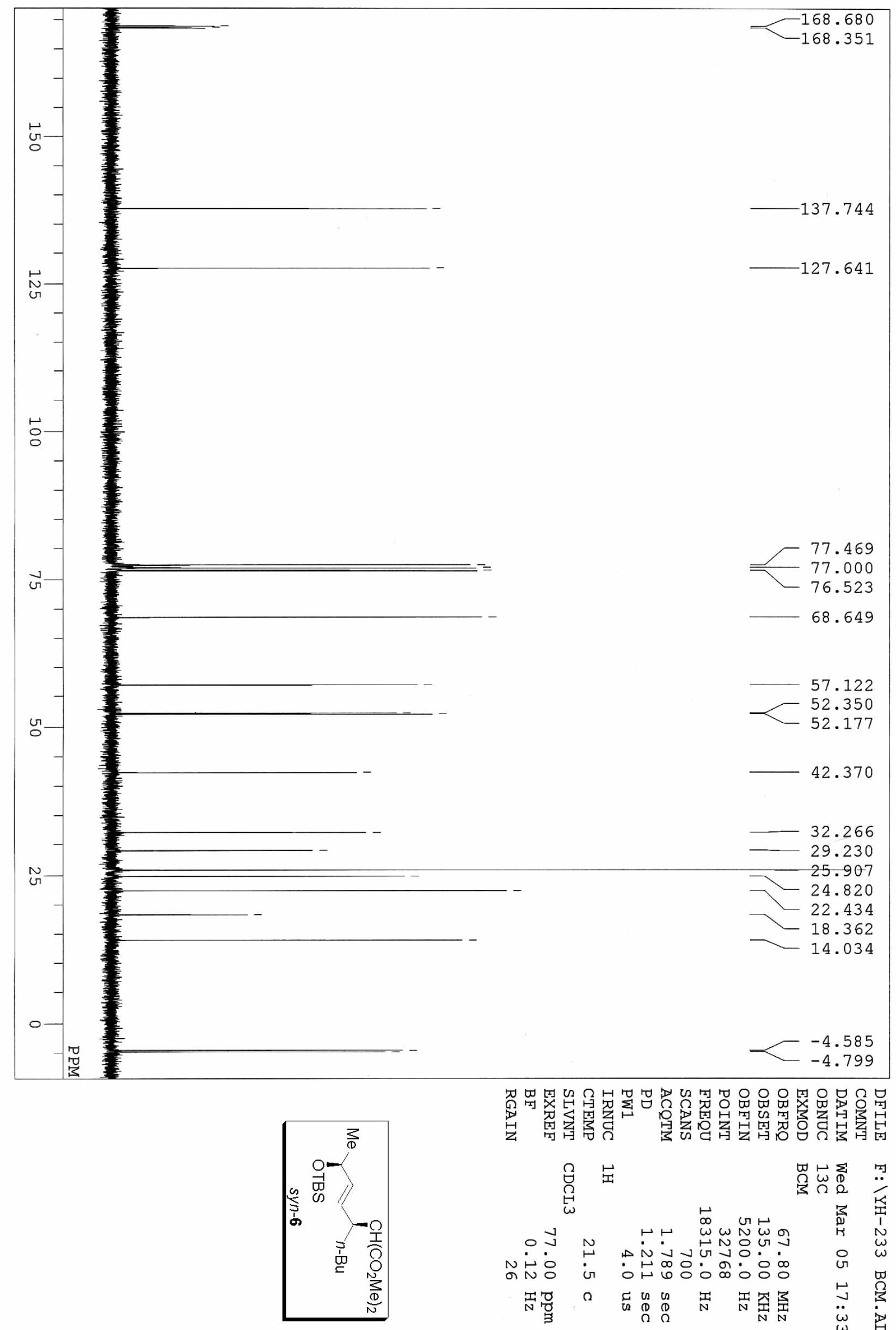

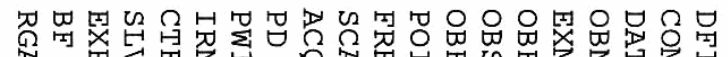

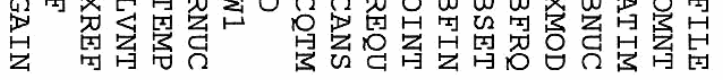

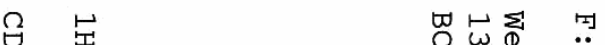

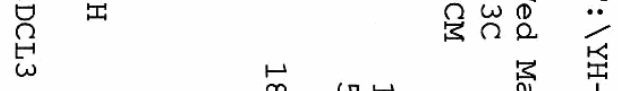
0.7 n N告客

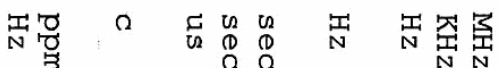




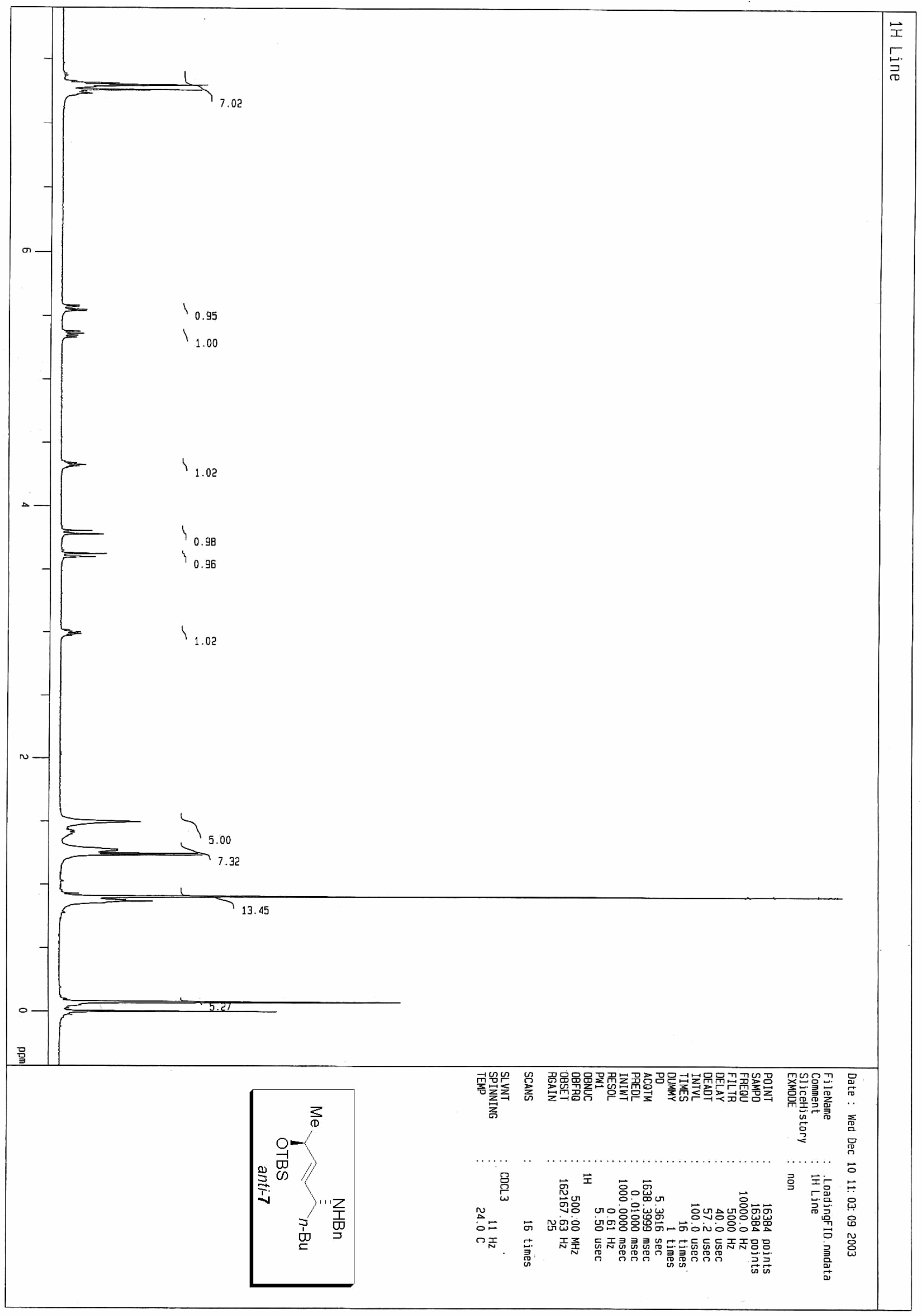




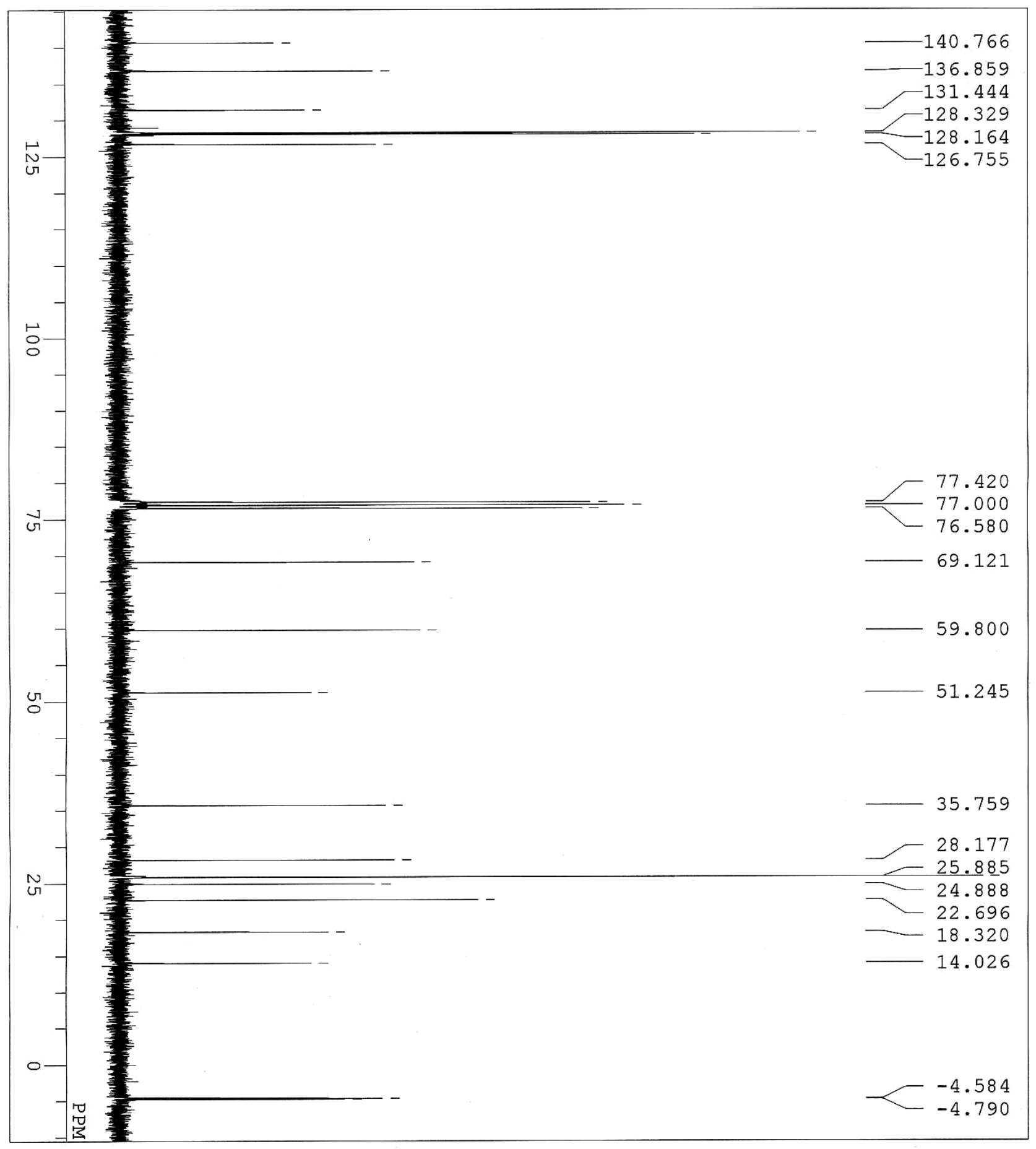

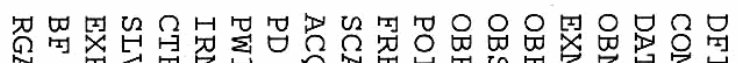

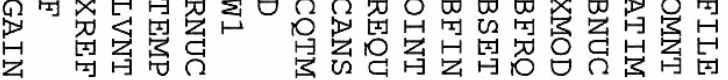

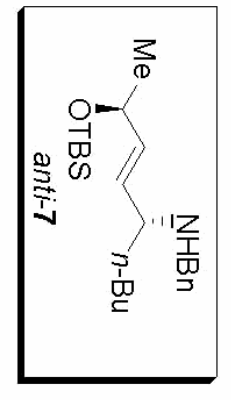

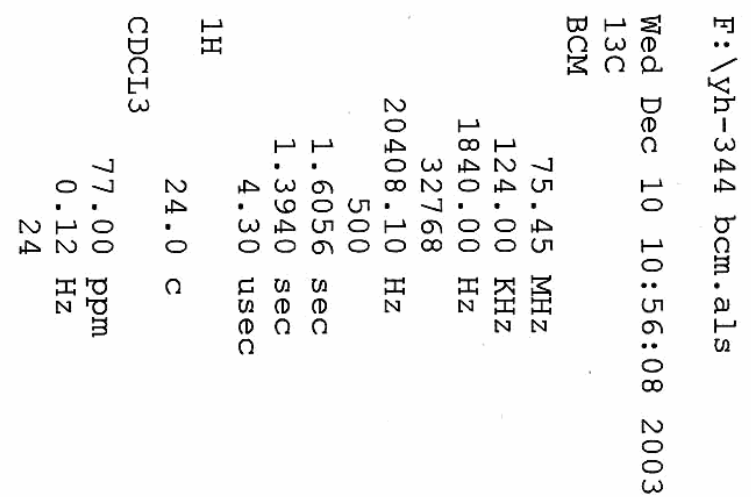




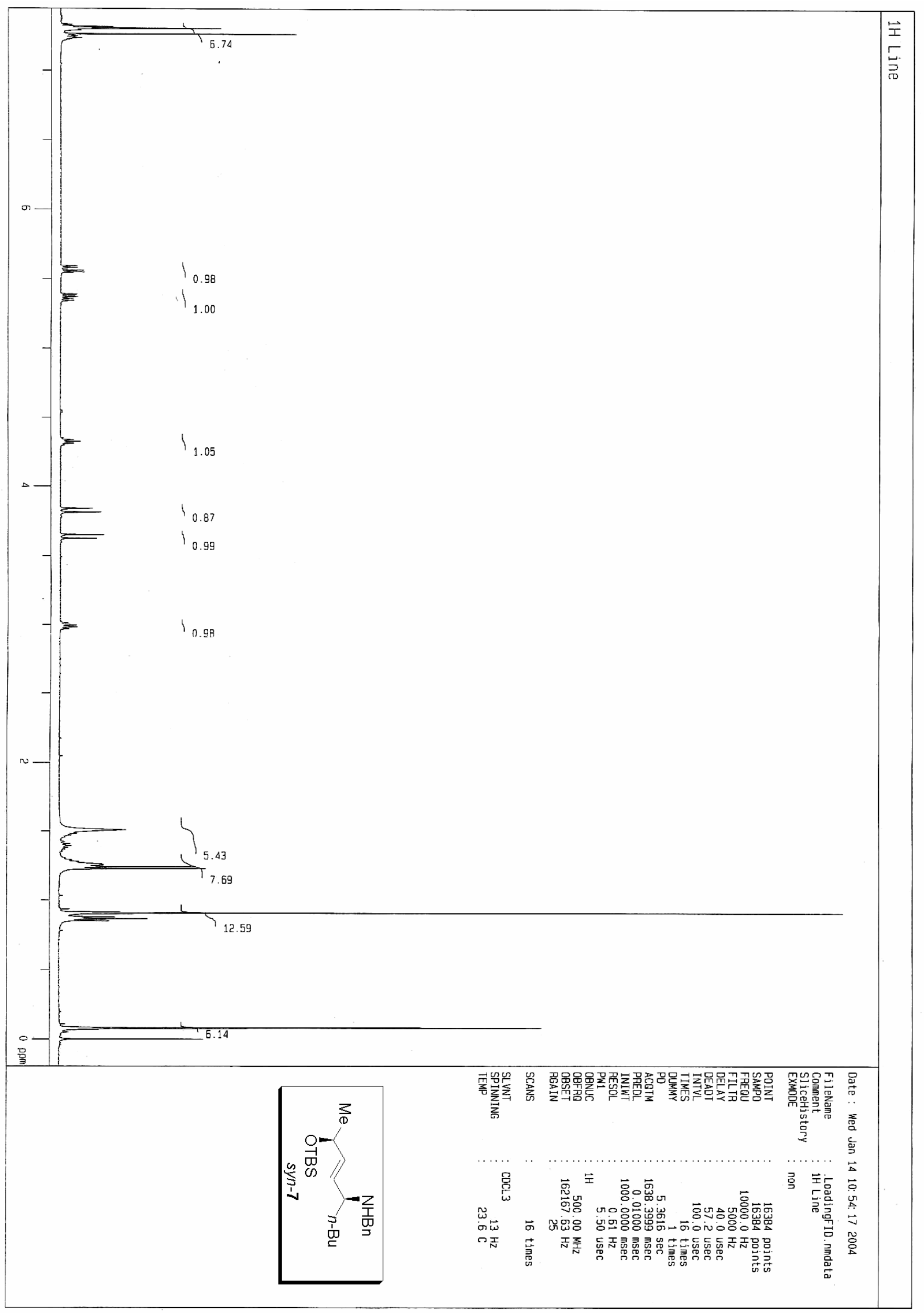




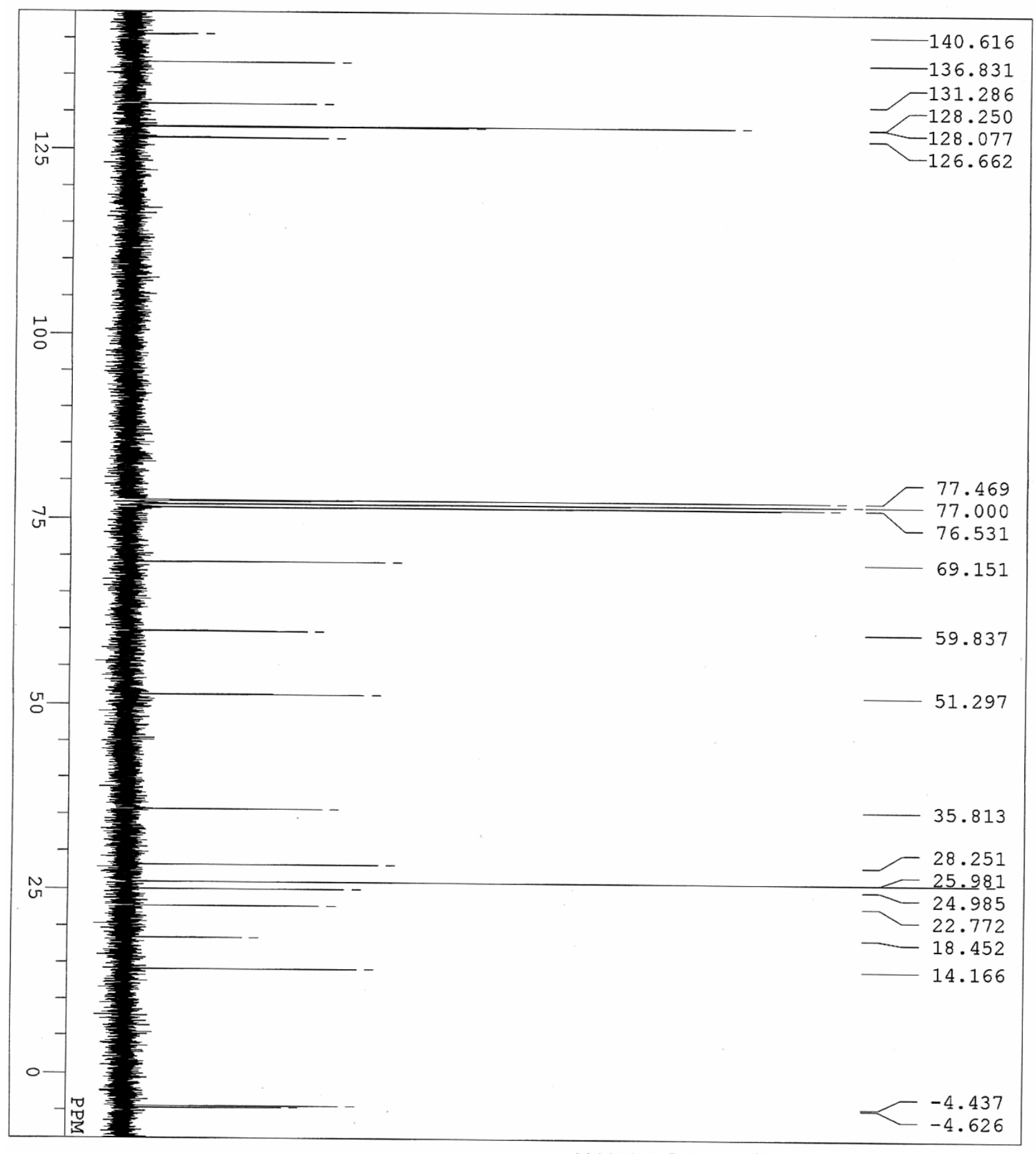

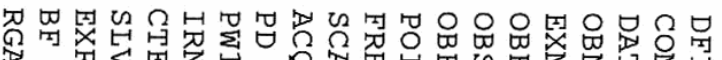

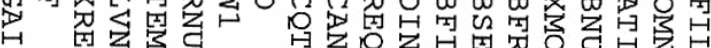

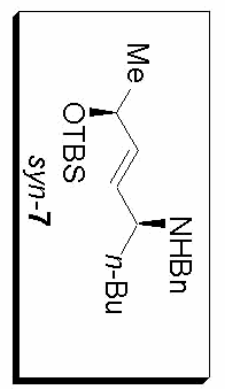

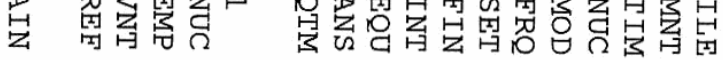

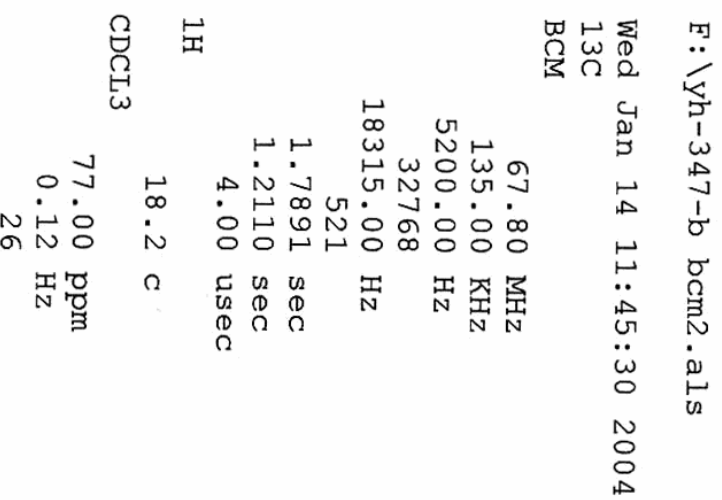




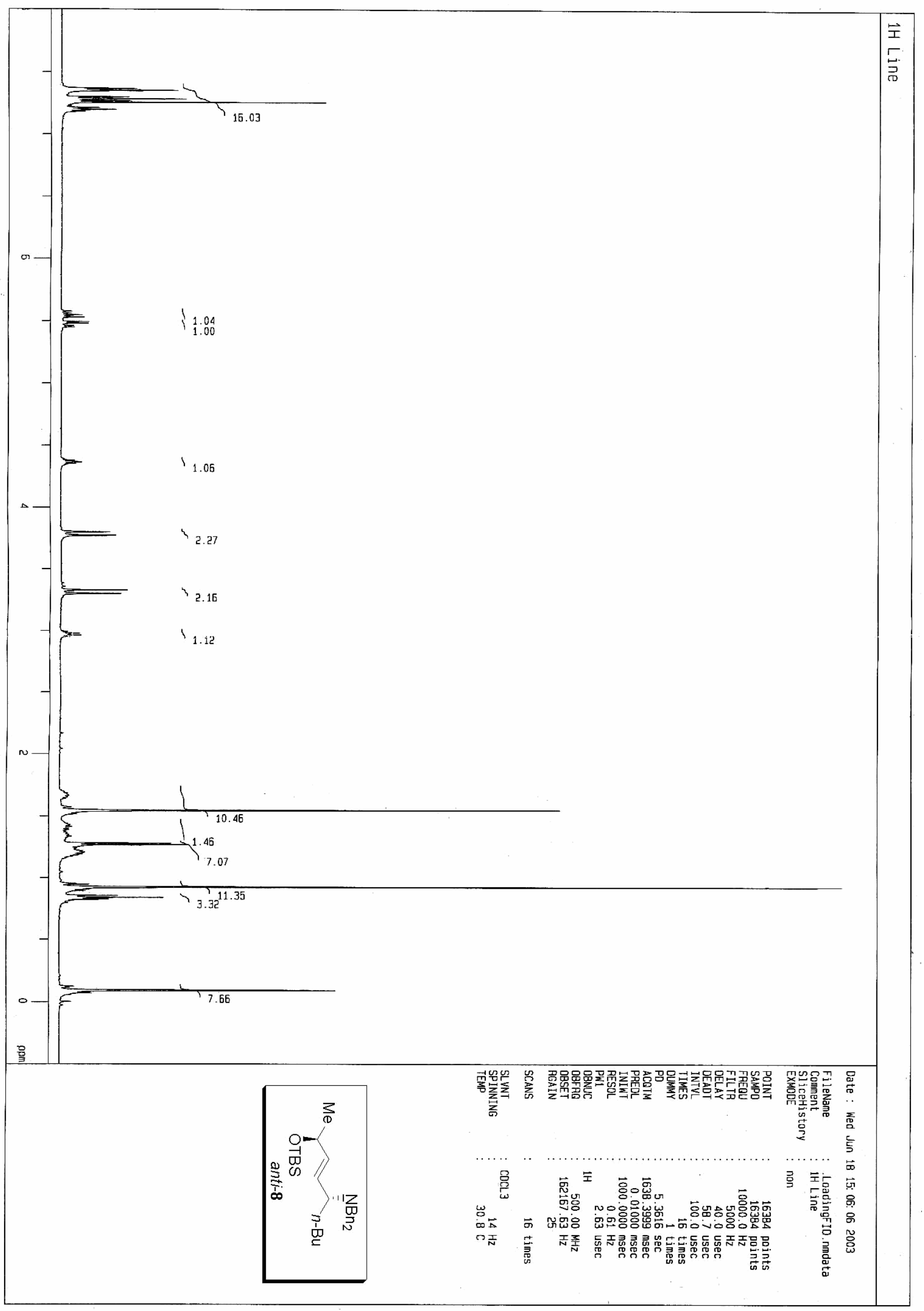




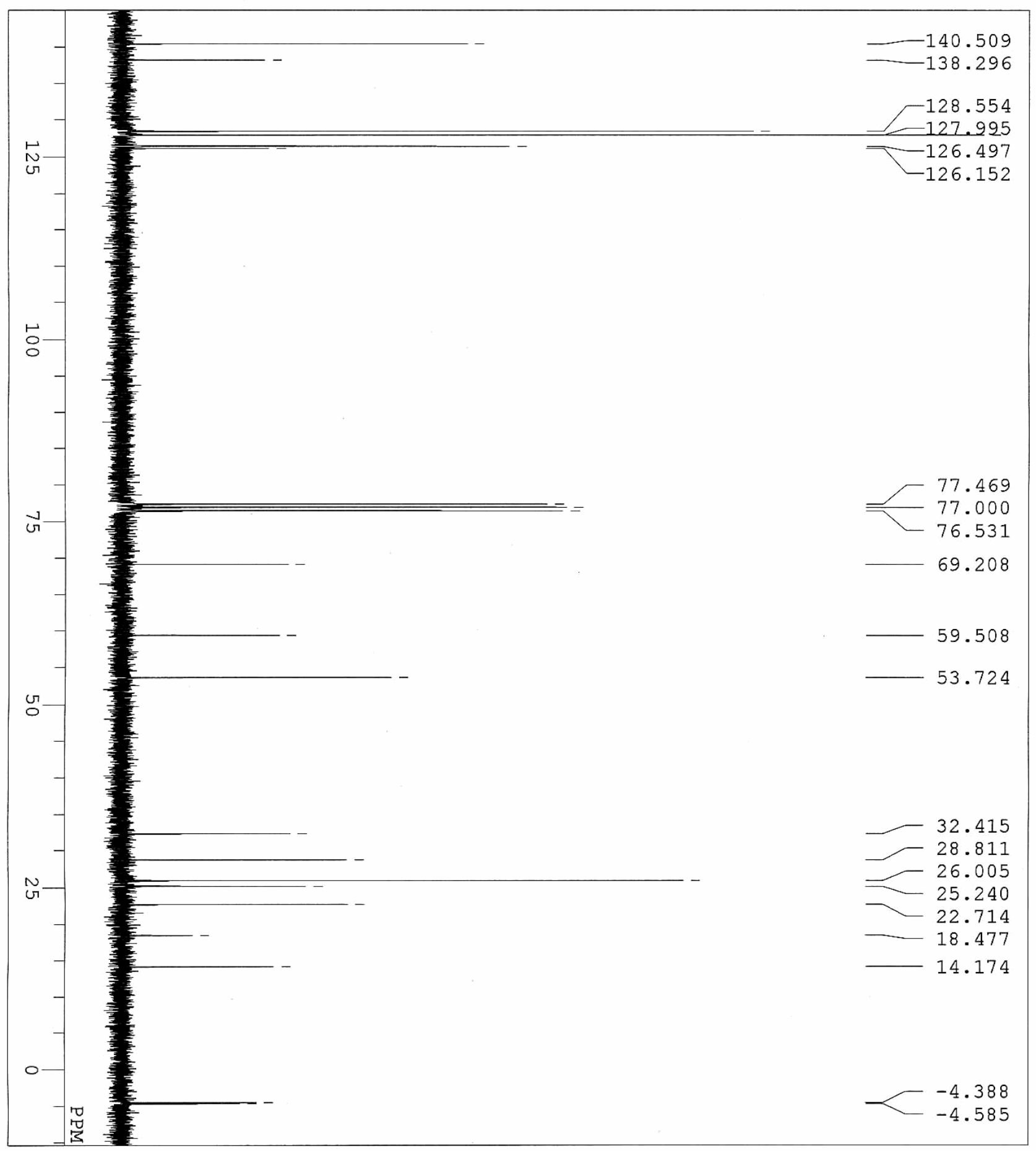

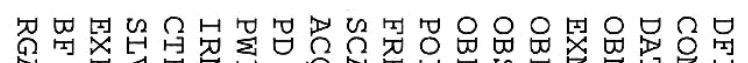

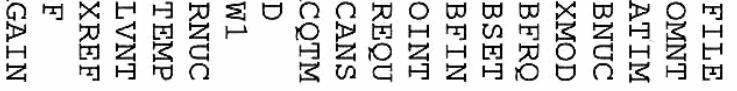

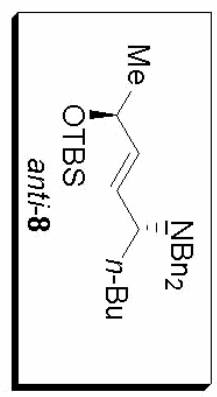

艺 品

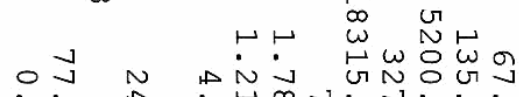

雚品宫

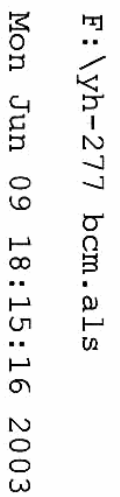




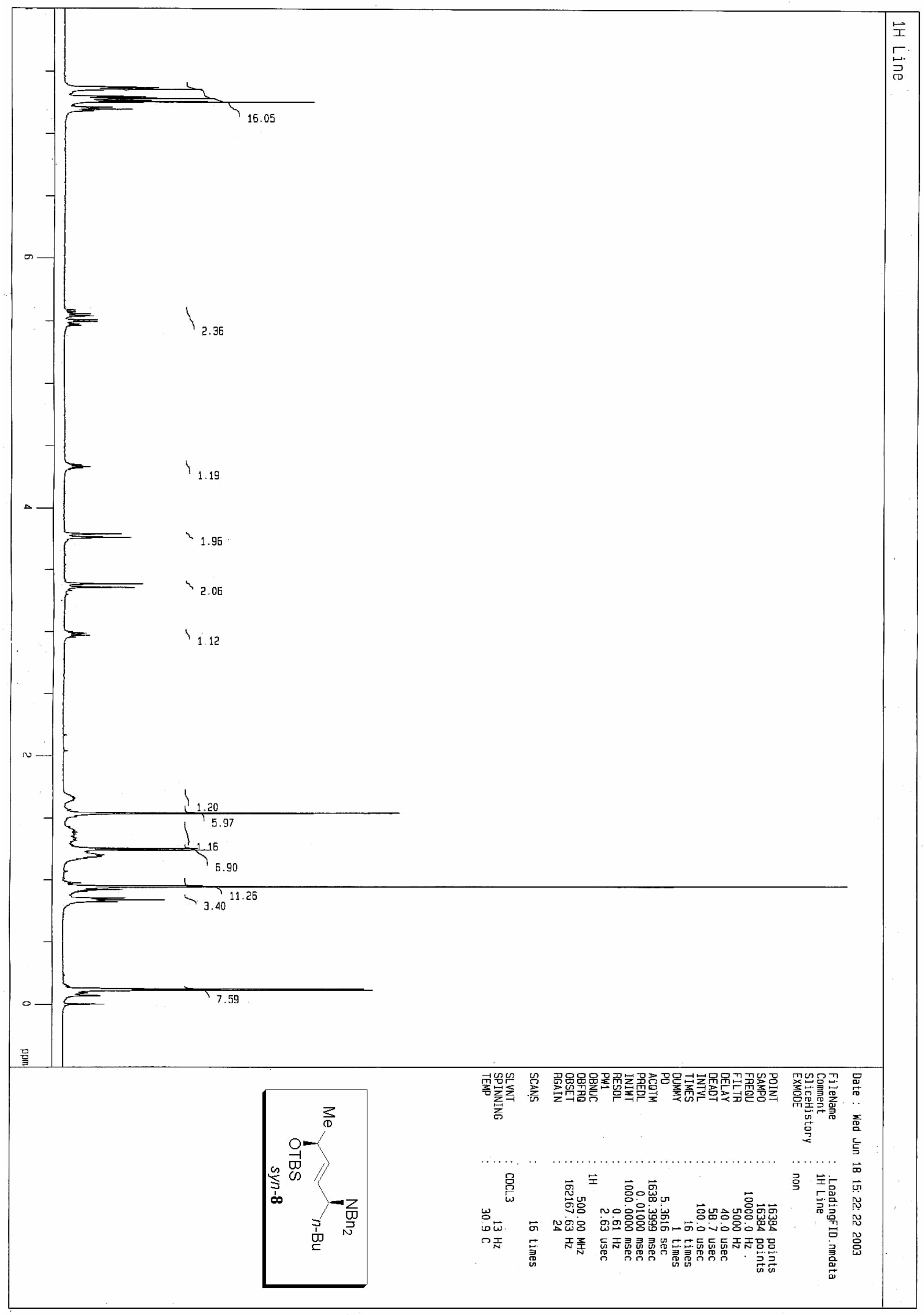

S12 


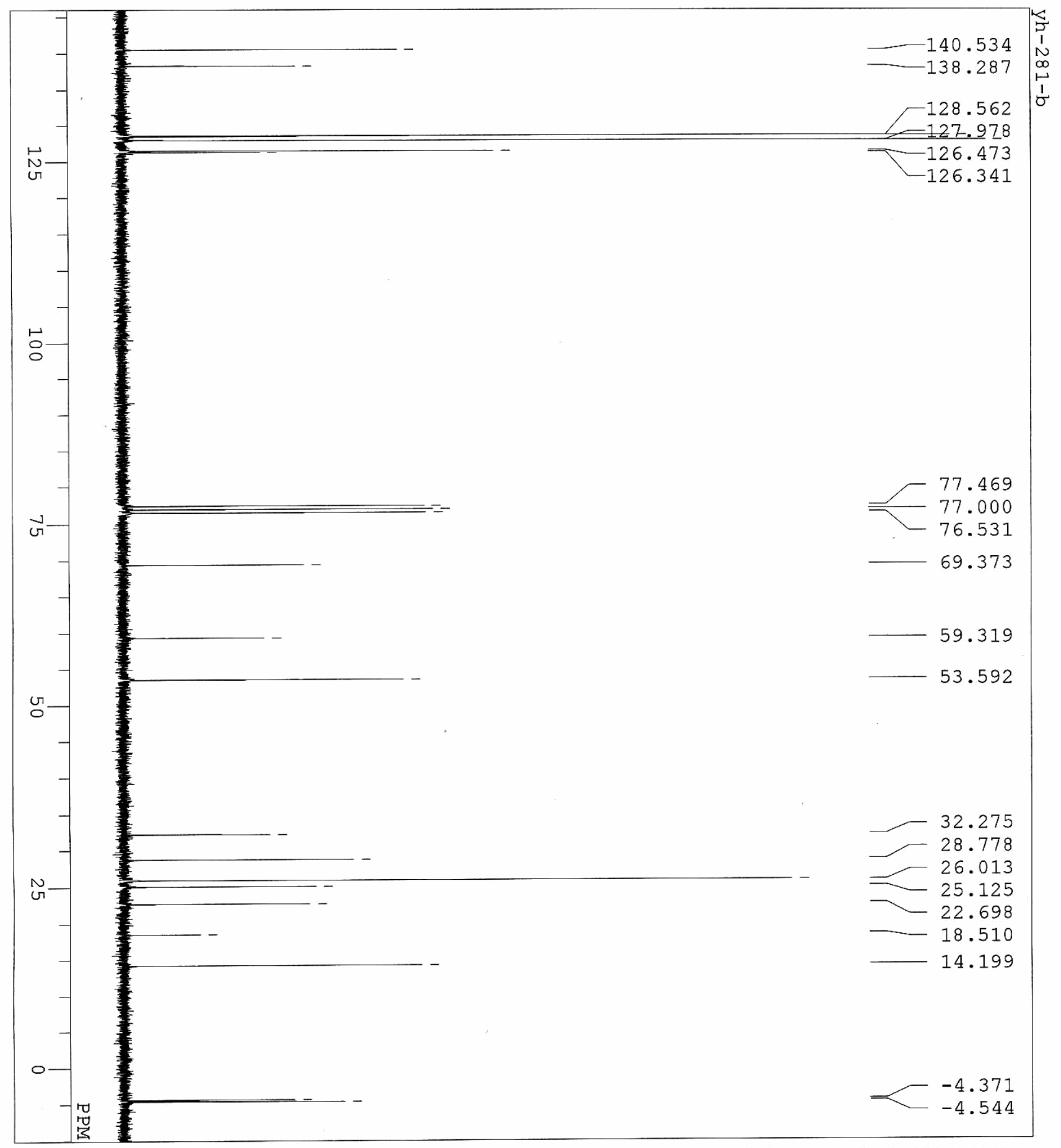

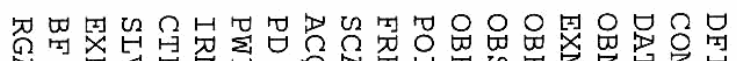

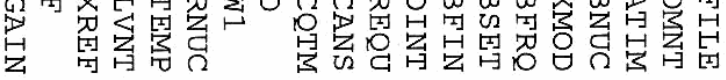
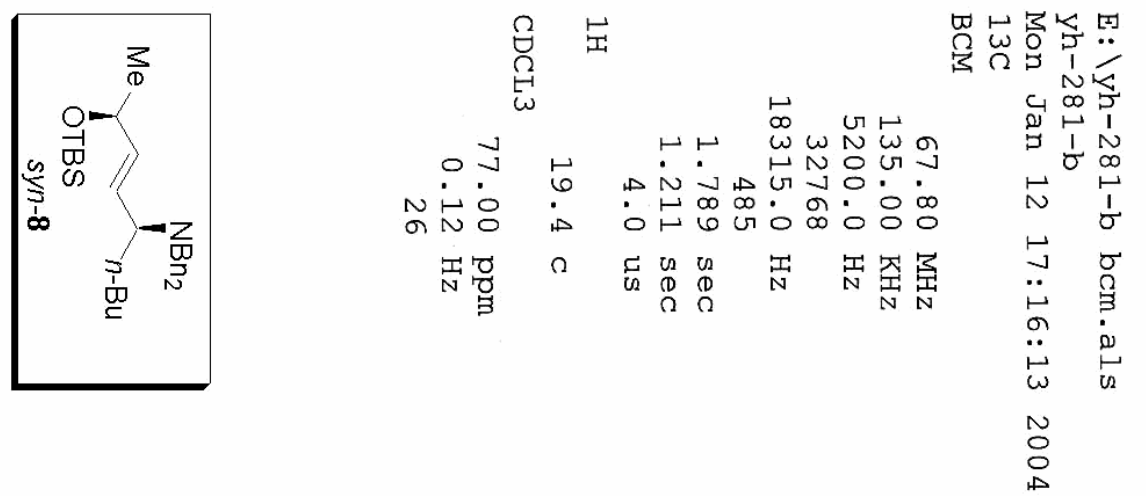


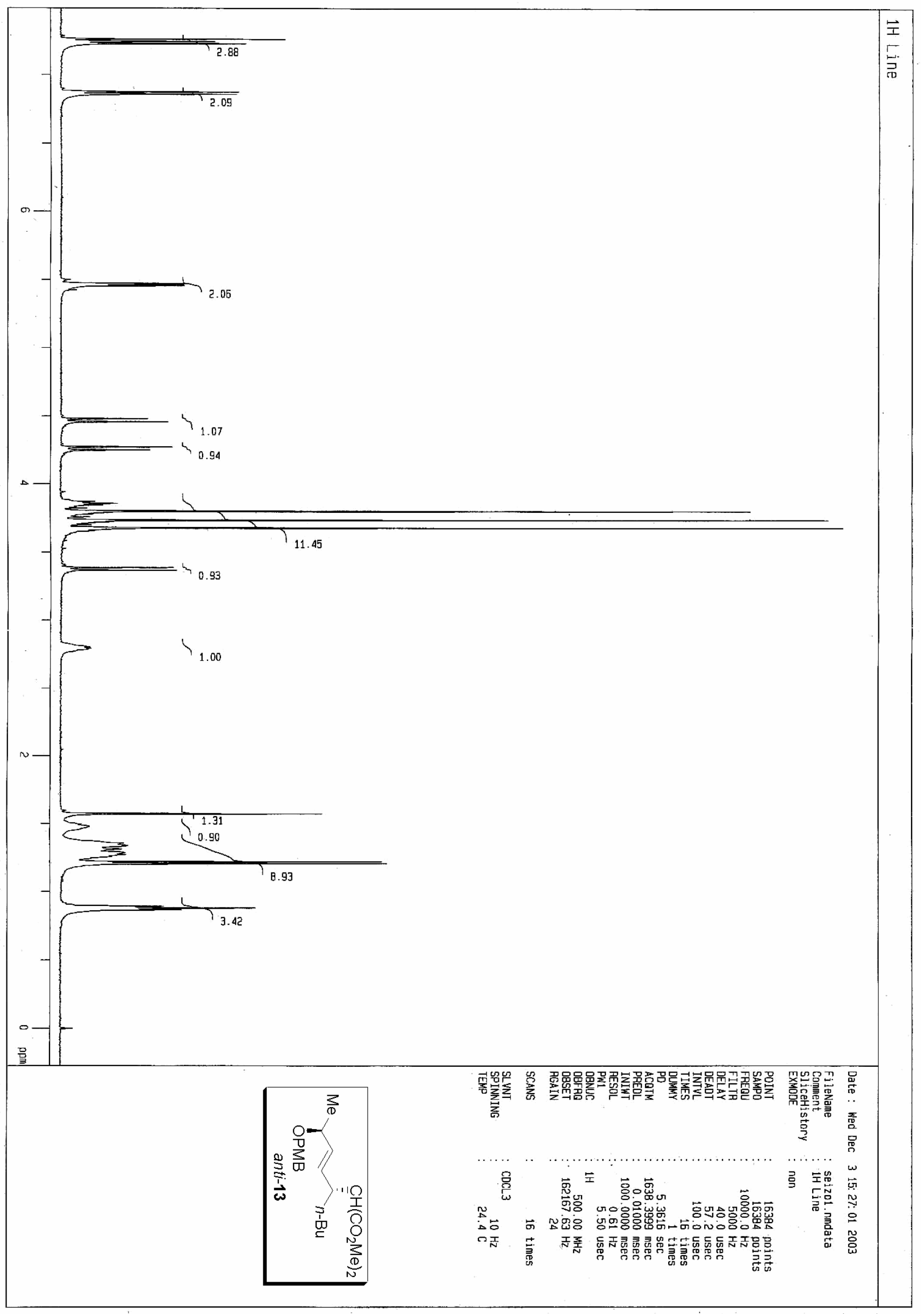



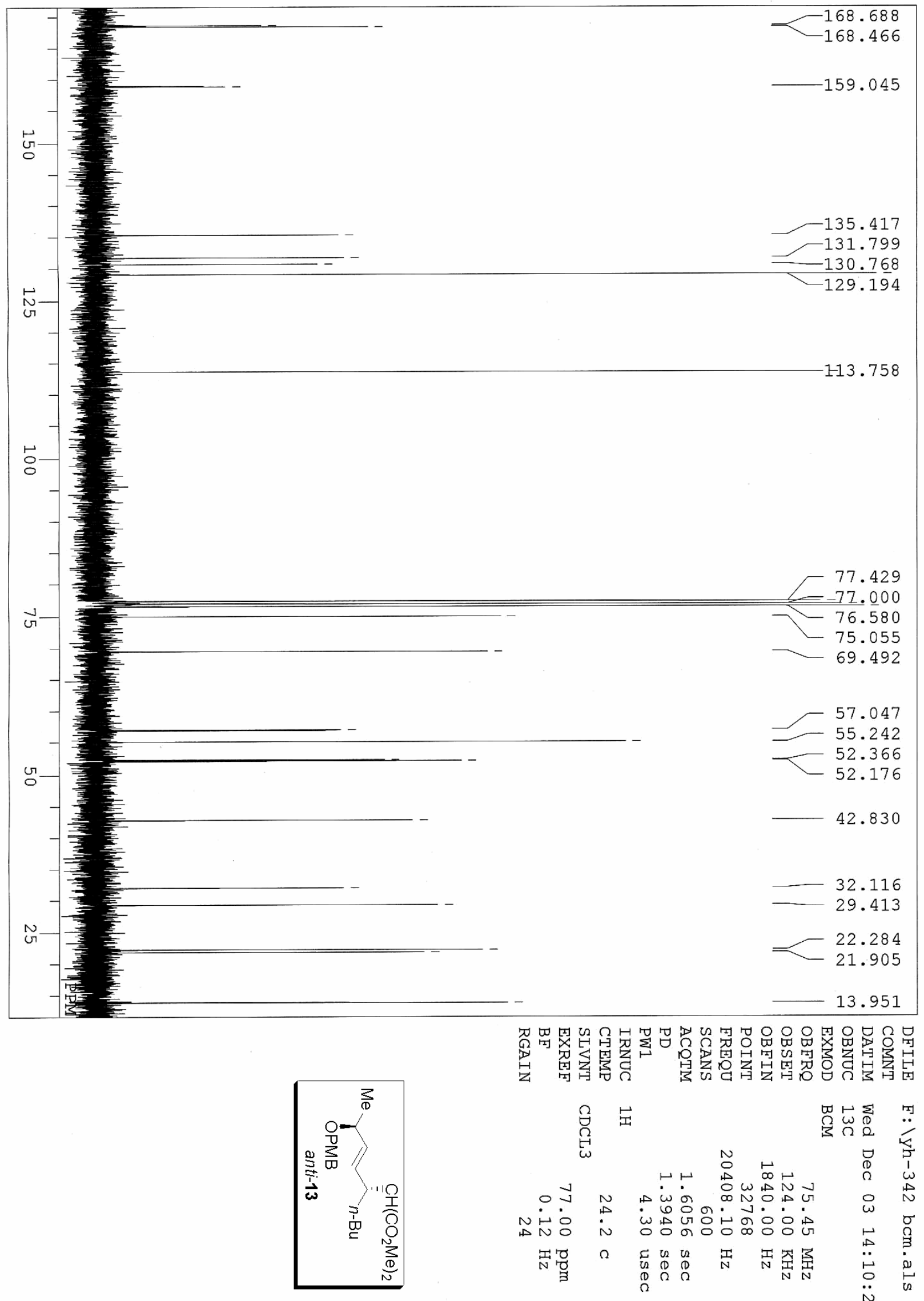

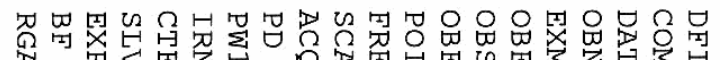

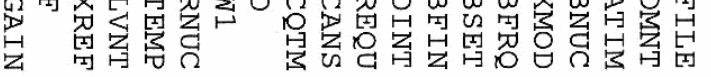<smiles>[BH3-][BH3-]</smiles>

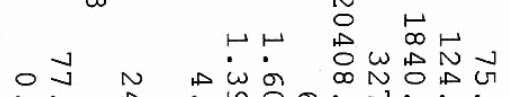

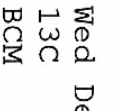

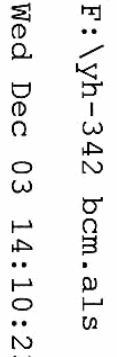

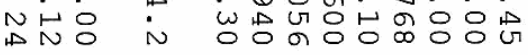

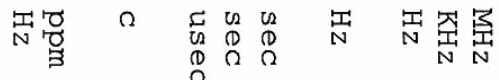

$\ddot{\omega}$

$N$
$\stackrel{\omega}{\circ}$
$\stackrel{\omega}{\omega}$ 


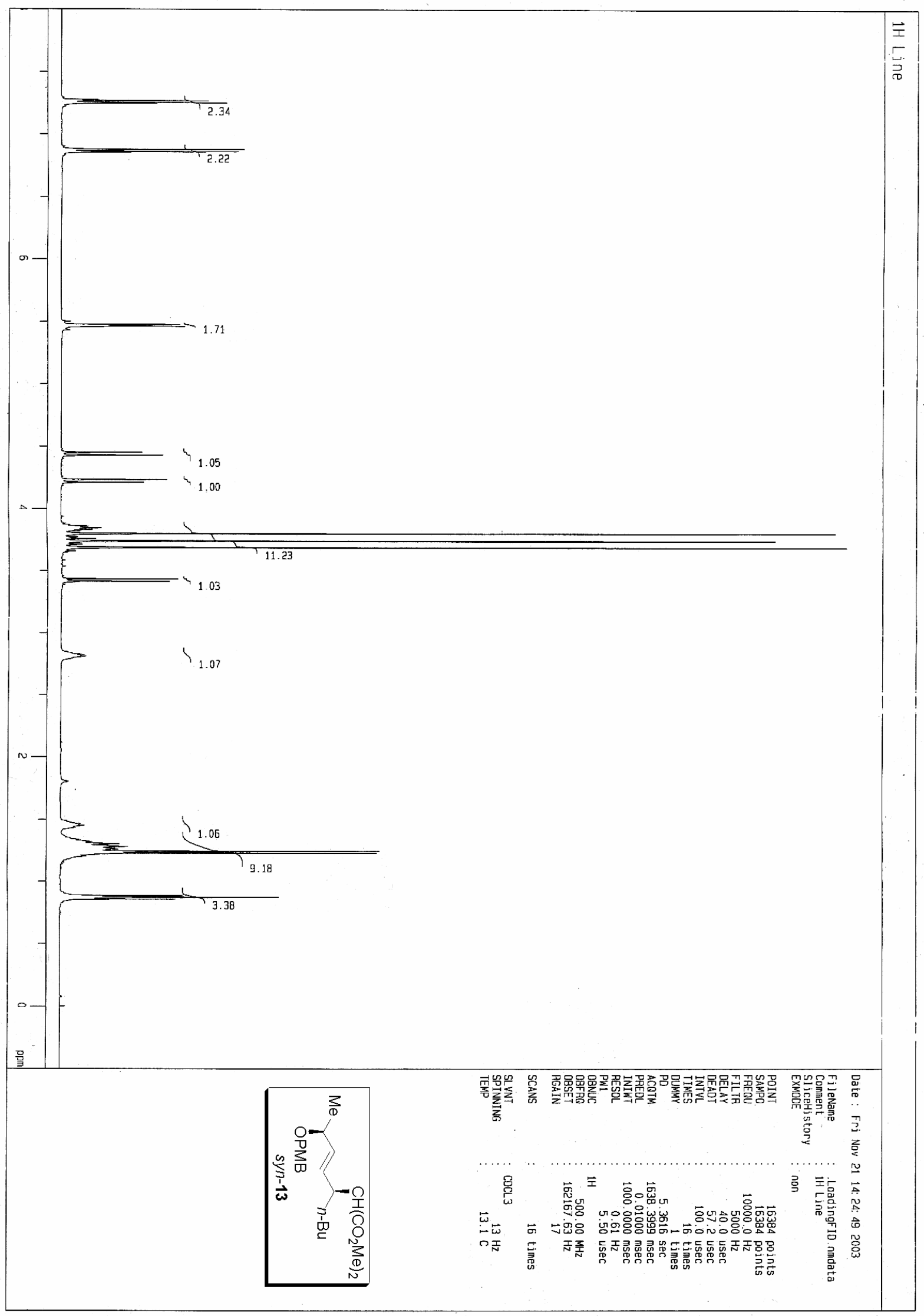




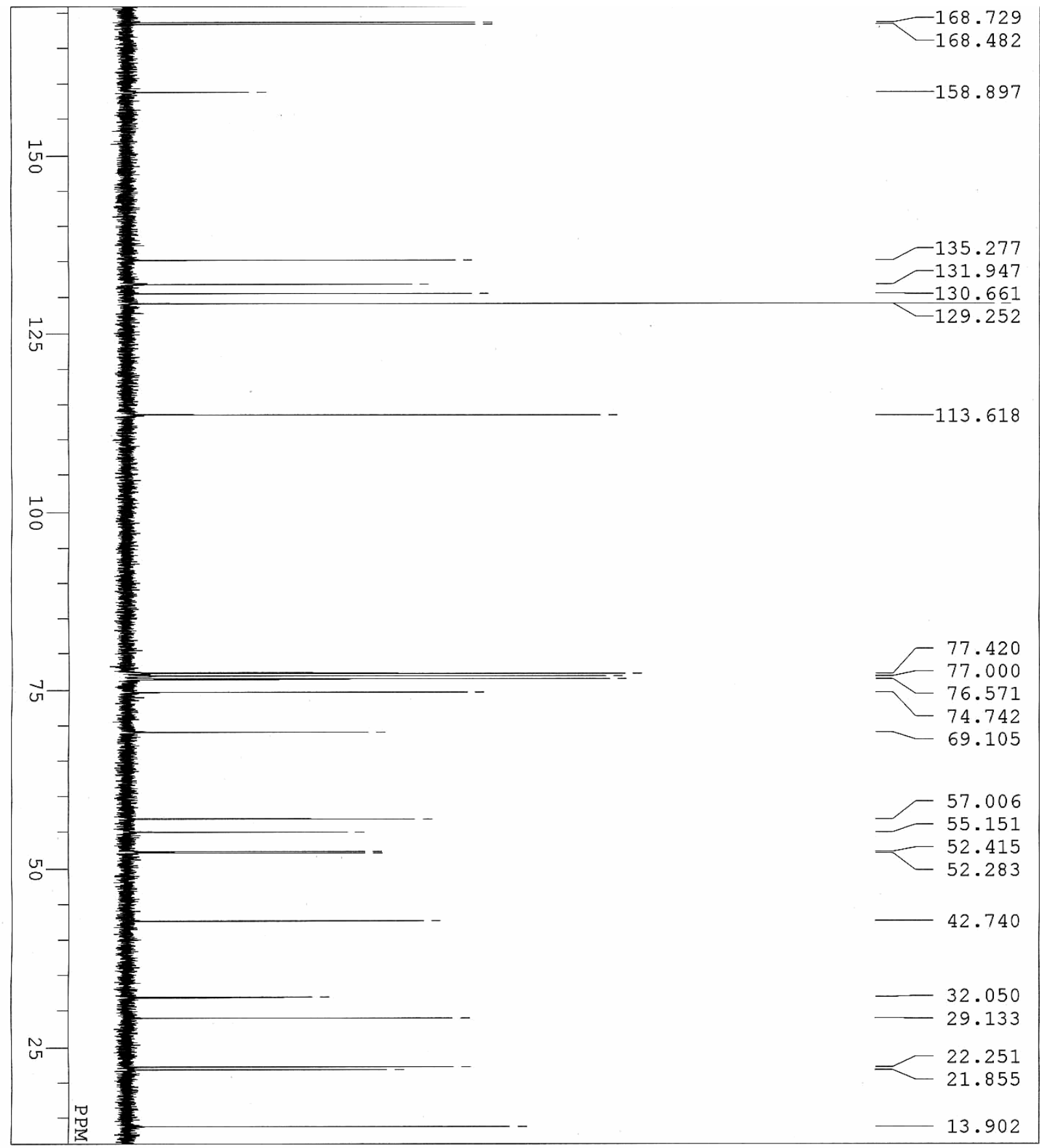

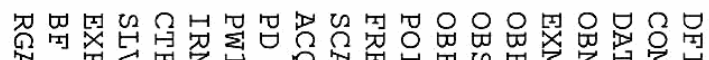

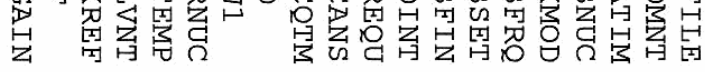

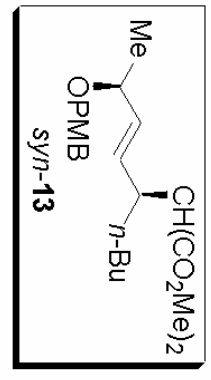

茵点

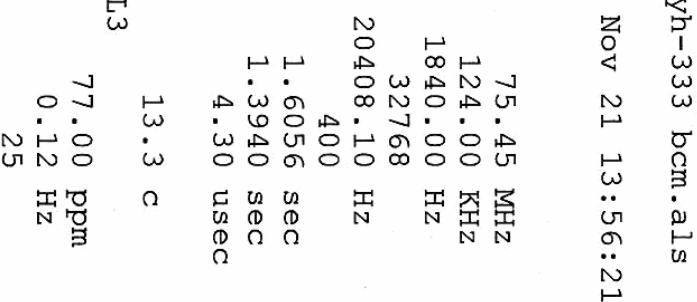

$\stackrel{N}{\circ}$ 


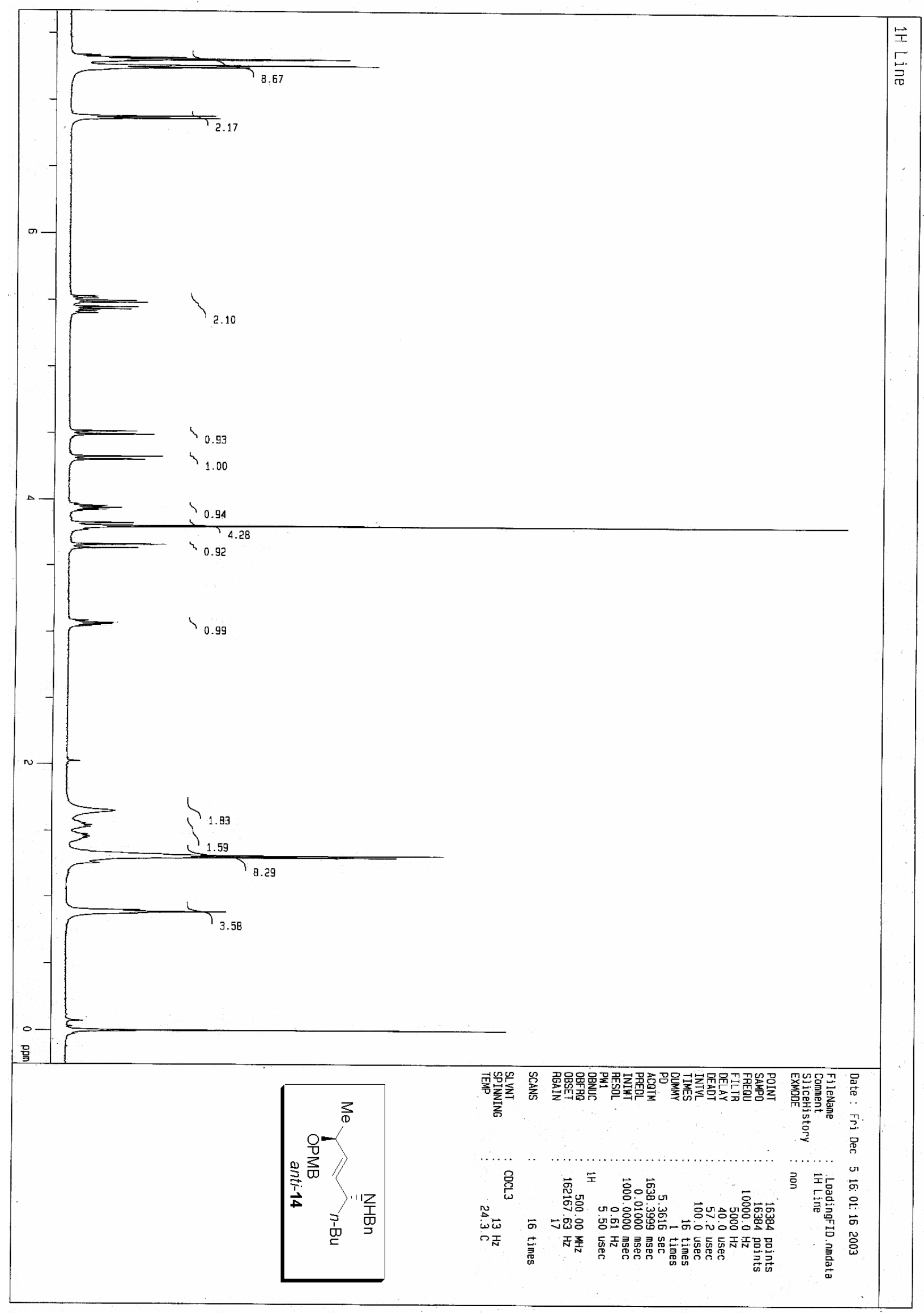




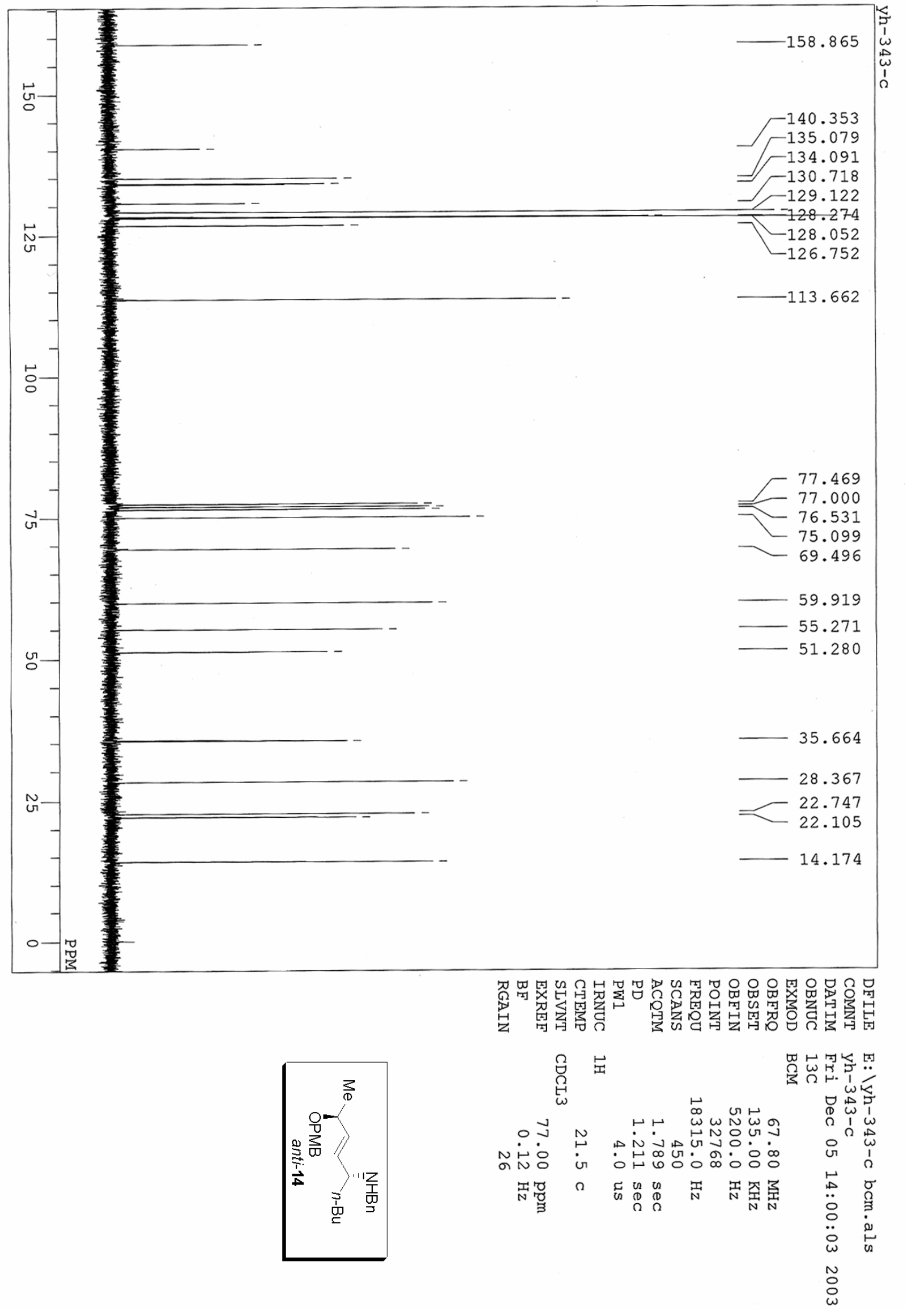




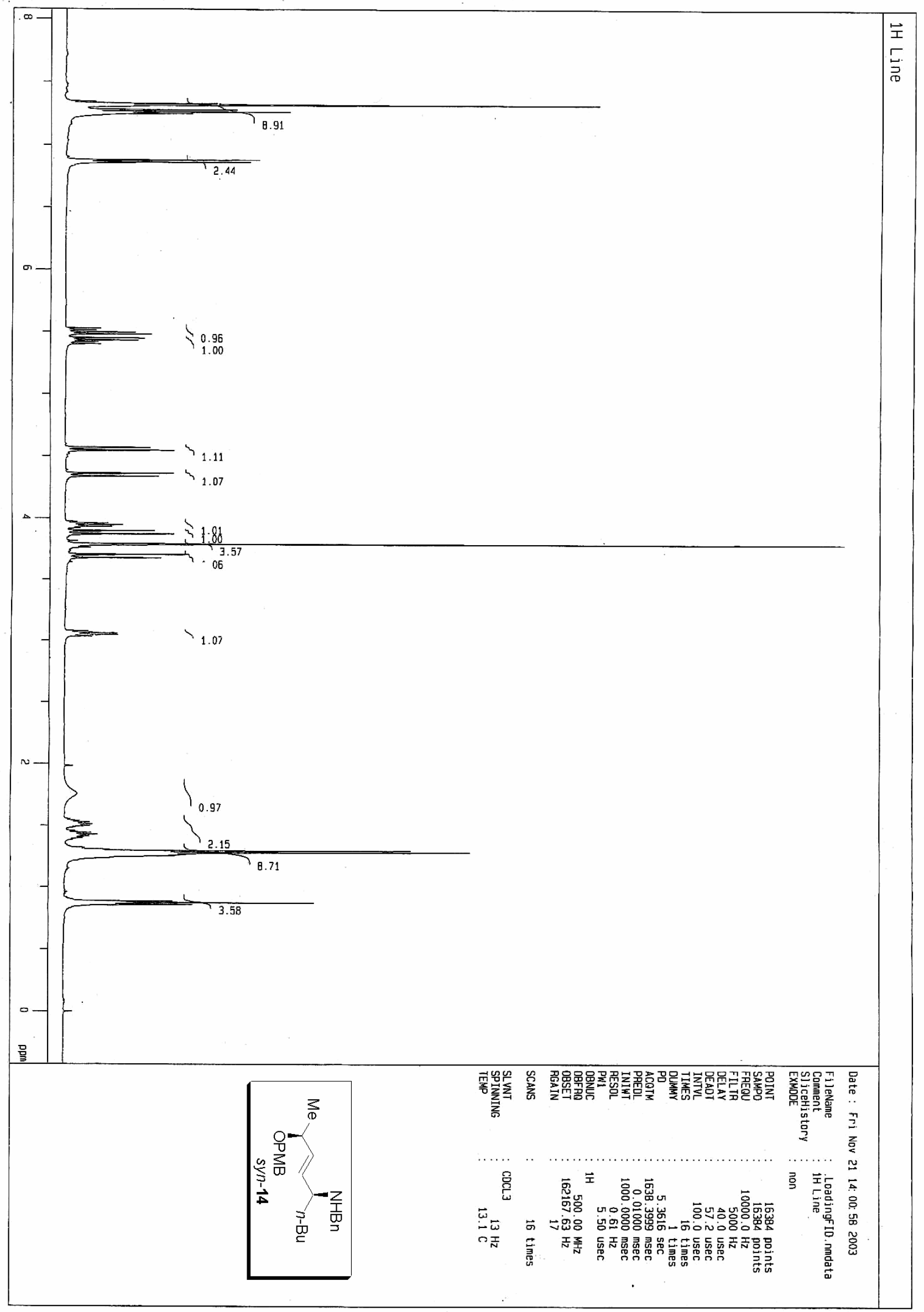




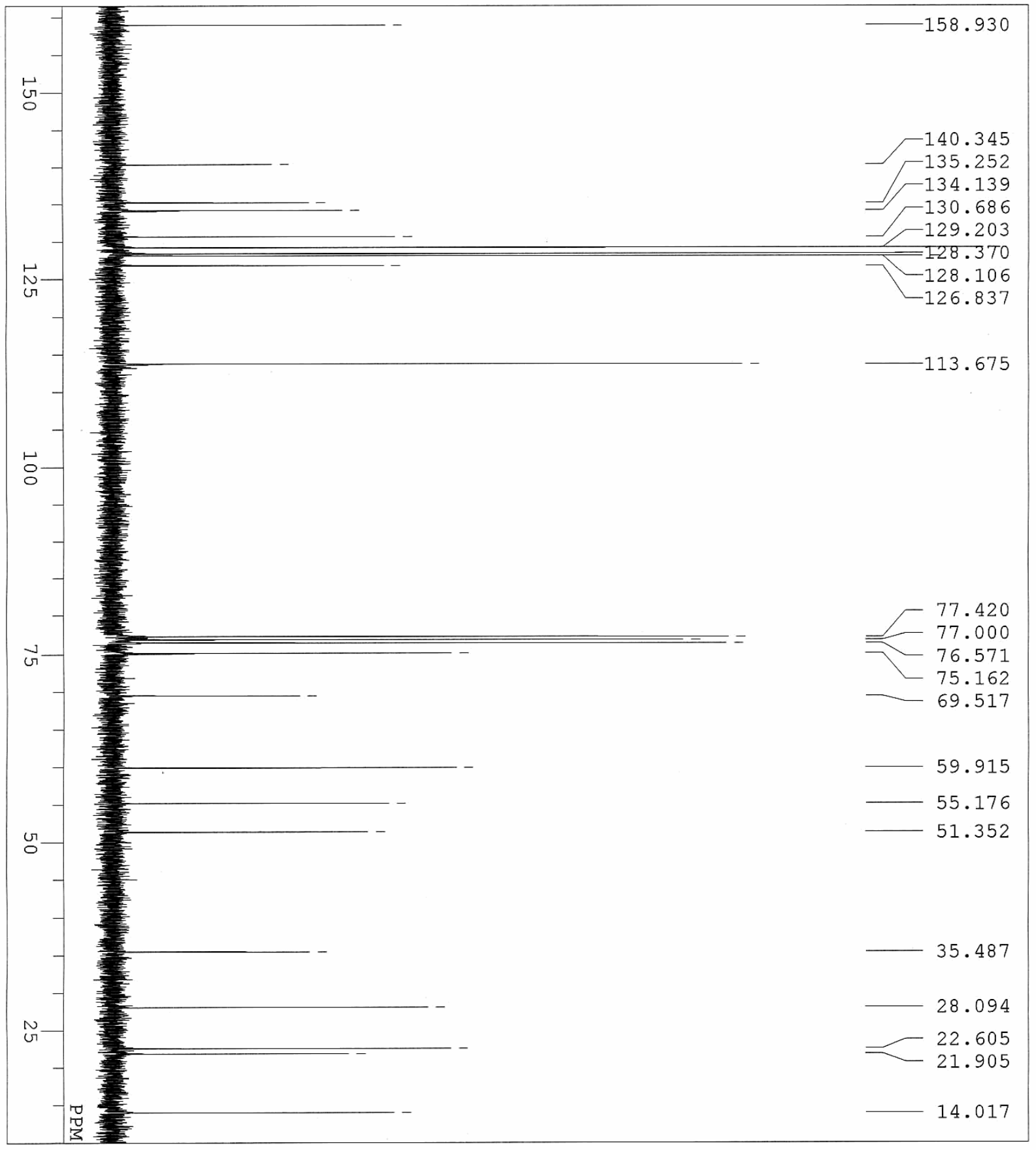

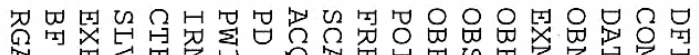

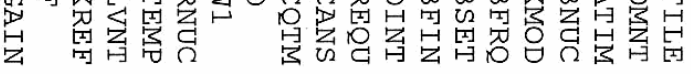

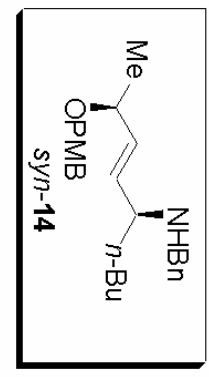<smiles>[Hg]=[Hg]</smiles>

崫空国

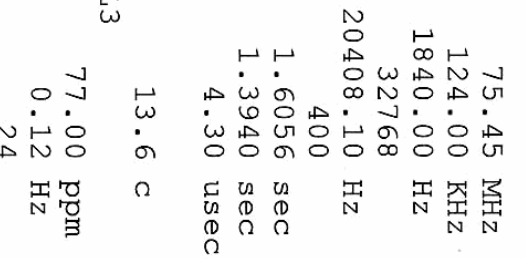

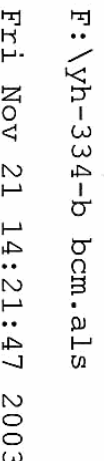

